\title{
Expression and prognostic significance of cancer-testis antigens (CTA) in intrahepatic cholagiocarcinoma
}

\author{
Jin-xue Zhou ${ }^{1+}$, Yin $\mathrm{Li}^{2+}$, Sun-xiao Chen ${ }^{3^{*}}$, An-mei Deng ${ }^{3^{*}}$
}

\begin{abstract}
Background: Cancer-testis antigens (CTAs) are suitable targets for cancer-specific immunotherapy. The aim of the study is to investigate the expression of CTAs in intrahepatic cholagiocarcinoma (IHCC) and evaluate their potential therapeutic values.

Methods: Eighty-nine IHCC patients were retrospectively assessed for their expression of CTAs and HLA Class I by immunohistochemistry using the following antibodies: MA454 recognizing MAGE-A1, 57B recognizing multiple MAGE-A (MAGE-A3/A4), E978 recognizing NY-ESO-1, and EMR8-5 recognizing HLA class I. The clinicopathological and prognostic significance of individual CTA markers and their combination were further evaluated.

Results: The expression rates of MAGE-A1, MAGE-A3/4 and NY-ESO-1 were 29.2\%, 27.0\% and 22.5\%, respectively. The concomitant expression of CTAs and HLA class I antigen was observed in $33.7 \%$ of the IHCC tumors. We found that positive MAGE-3/4 expression correlated with larger tumor size $(\geq 5 \mathrm{~cm})$, tumor recurrence and poor prognosis. Moreover, we identified 52 cases (58.4\%) of IHCC patients with at least one CTA marker expression, and this subgroup displayed a higher frequency of larger tumor size and a shorter survival than the other cases. Furthermore, expression of at least one CTA marker was also an independent prognostic factor in patients with IHCC.
\end{abstract}

Conclusion: Our data suggest that specific immunotherapy targeted CTAs might be a novel treatment option for IHCC patients.

\section{Introduction}

Intrahepatic cholagiocarcinoma (IHCC) is a relatively uncommon malignancy, comprising approximately 5\%$10 \%$ of the liver cancers, and both its incidence and mortality have increased in recent years in China and other countries $[1,2]$. IHCC is not sensitive to radiation therapy and chemotherapy. Even the patients undergoing a radical surgical resection is still at a high risk for early recurrence, and the patients' survival is thus unsatisfactory. Therefore, there is a great need to identify molecular targets for developing novel therapeutic approaches for patients with IHCC.

Cancer testis antigens (CTAs) comprise a group of non-mutated self-antigens selectively expressed in

\footnotetext{
* Correspondence: chensunxiao@126.com; anmeideng@yahoo.com.cn + Contributed equally

${ }^{3}$ Changzheng Hospital, Second Military Medical University, Shanghai 200003, PR China

Full list of author information is available at the end of the article
}

various tumors and normal testis tissues, but not in other normal tissues [3]. Several studies have shown that if presented with human leukocyte antigen (HLA) class I molecules, these tumor-associated antigens could induce effective anti-tumor cytotoxic $\mathrm{T}$ lymphocytes (CTLs) response in vitro and in vivo [4]. Because of these unique characteristics, CTAs are regarded as promising targets for cancer-specific immunotherapy [5]. However, the possibility that IHCC patients might benefit from CTA-targeted therapies has not been evaluated.

Given their potential therapeutic significance, it may have significance for exploring the presence of CTAs in IHCC. However, to our knowledge, until now, only two studies examined the mRNA and protein expression of CTAs in small number of IHCC cases [6,7]. The CTAs expression at protein level and their clinicopathological and prognostic significance in a larger cohort have not been investigated.

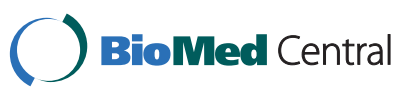

(c) 2011 Zhou et al; licensee BioMed Central Ltd. This is an Open Access article distributed under the terms of the Creative Commons Attribution License (http://creativecommons.org/licenses/by/2.0), which permits unrestricted use, distribution, and reproduction in any medium, provided the original work is properly cited. 
The aims of the current study were to analyze the expression of MAGE-A1, MAGE-A3/4 and NY-ESO-1 CTAs in IHCC tissues by immunohistochemistry, and to investigate correlations between their expression with HLA class I expression, clinicopathologic parameters and survival in patients with IHCC.

\section{Materials and methods Patients}

The study was approved by the research ethics committee of our institutions, and informed consent was obtained from each patient. A total of consecutive 102 patients with IHCC who underwent curative resection at Department of Hepatobiliary and Pancreatic Surgery, Henan Tumor Hospital (Zhengzhou, China) and Changzheng Hospital (Shanghai, China) from 1999 to 2006 were retrospectively reviewed. Patients with lymphnode-positive metastasis routinely received 5-fluorouracil-based chemotherapy, and Gemcitabone chemotherapy was given when recurrence occurred. Patients were followed up every two month during the first postoperative year and at every four month afterward. Follow-up was finished on May 2008. The median follow-up was 24 month (range, 4-61 month). Overall survival (OS) time was defined as the time from operation to cancer-related death only.

Cases were included according to the following inclusion criteria: having archived formalin-fixed, paraffin-embedded specimens available; having complete clinicopathological and followed-up data; receiving no anticancer treatment before operation. Patients who died of unrelated diseases and within one month after operation were excluded, leaving 89 patients eligible for this analysis. The clinical and pathological details of these patients were summarized in Additional file 1.

\section{Immunohistochemical analysis}

Immunohistochemical analysis was performed on archived tissue blocks containing a representative fraction of the tumors. Briefly, 5- $\mu$ m-thick paraffinembedded tissue sections were deparaffinized and rehydrated. Endogenous peroxidase was blocked with methanol and $0.3 \% \mathrm{H}_{2} \mathrm{O}_{2}$ for $20 \mathrm{~min}$. Antigen retrieval was performed with microwave treatment in $0.1 \mathrm{M}$ sodium citrate buffer ( $\mathrm{pH}$ 6.0) for $10 \mathrm{~min}$. Expression of CTAs was detected with the monoclonal antibody against MAGE-A1 (clone MA454), MAGE-A3/4 (clone 57B) and NY-ESO-1 (clone E978), as described previously [8-10]. Clone 57B was originally raised against MAGE-A3, and later has been reported to primarily recognize the MAGE-A4 antigen [11,12]. Currently, 57B is considered to be anti-pan-MAGE-A (MAGE-A3/4). Expression of HLA class I was detected with an antipan HLA class I monoclonal antibody EMR8-5, as described previously [13]. Detection was performed with the Dako Envision system using diaminobenzidine (DAB) as the chromogen. Non-specific mouse IgG was used as negative control and normal human testis tissues were used as positive controls for CTA expression. Immunochemical results were evaluated and scored by two and independent observers according to the previous criteria [14]. Positive CTA expression was assigned to any extent of immunostaining in sections and further graded into four groups: $+:<5 \%$ of tumor cells stained; $++: 5-25 \%$ of tumor cells stained; $+++:>25-50 \%$ of tumor cells stained; $++++:>50 \%$ of tumor cells stained. A patient was considered CTA-positive if at least one of three markers demonstrated positive immunoactivity. HLA class I expression was classified as positive and down-regulated compared with stromal lymphocytes as an internal control as previously described [13].

\section{Statistical analysis}

The associations between CTAs expression and clinicopathological parameters were evaluated using Chi-square or Fisher's exact test, as appropriate. Overall survival of patients were estimated by the Kaplan-Meier method, differences between groups were compared were by the log-rank test. Multivariate analysis was performed using a Cox proportional hazard model. Statistically significant prognostic factors identified by univariate analysis were entered in the multivariate analysis. All the statistical analyses were performed with SPSS 16.0 software. $P$ value less than or equal to 0.05 was considered statistically significant.

\section{Results}

Expression of MAGE-A1, MAGE-A3/4, NY-ESO-1 and HLA class I proteins in IHCC patients by immunohistochemistry

MAGE-A1, MAGE-A3/4 and NY-ESO-1 showed a predominantly, although not exclusively, cytoplasmic staining (Figure 1). The frequency and grade of various CTA expressions in tumors is shown in Table 1. Figure 2 showed a Venn diagram dipicting the overlap of three CTAs expression. When the CTA combinations were tested, 52 from 89 IHCC cases (58.4\%) showed expression of at least one marker, 14 cases (15.7\%) demonstrated co-expression of two CTAs, and only three cases $(3.3 \%)$ were positive for all the three antigens. As seen in table 2, down-regulated HLA class I expression was found in $42.7 \%$ of all tumors $(n=38)$. Comparing the relationship between individual or combined CTAs expression and HLA-class I expression, no correlation was observed. And 30 IHCC cases (33.7\%) demonstrated concomitant expression of CTAs and HLA class I antigen. 


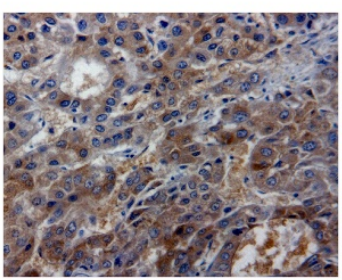

A

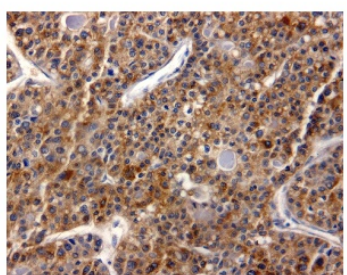

C

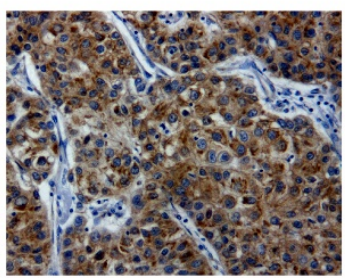

B

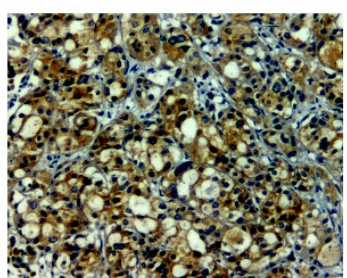

D
Figure 1 Immunohistochemical analysis of MAGE-A1, MAGEA3/ 4, NY-ESO-1 and HLA Class I in intrahepatic cholagiocarcinoma. Sections were stained with antibody against (A) MAGE-A1 (MA454): (B) MAGE-A3/A4 (57B); (C) NY-ESO-1 (E978); (D) HLA Class I (EMR8-5).

\section{Correlation between CTAs expression with HLA-class I}

\section{expression and clinicopathological parameters}

We found that positive MAGE-A3/4 and one CTA marker expression were detected more frequently in tumors with bigger size $(\geq 5 \mathrm{~cm})(20 / 24,38 / 52)$, than in smaller tumors $(\mathrm{P}=0.011, \mathrm{P}=0.009)$. In addition, MAGE-A3/4 positive IHCC had a higher recurrence rate $(17 / 24)$ than negative subgroup $(30 / 65, \mathrm{P}=0.038)$. There was no statistically significant correlation found between individual or combined CTA expression and any other clinicopathological traits.

\section{Correlation between CTAs expression and overall survival} The correlation of clinicopathological parameters and individual or combined CTA expression with overall survival was further investigated. As shown in Table 3, univariate analysis showed that overall survival significantly correlated with TNM stage, lymphnode metastasis, resection margin, differentiation and tumor recurrence but not with gender, age, tumor size and number, vascular invasion and perineural invasion.

Table 1 Expression of cancer-testis antigens in intrahepatic cholanglocarcinoma

\begin{tabular}{clll}
\hline & MAGE-A1 N (\%) & MAGE-A3/4 N (\%) & NY-ESO-1 N (\%) \\
\hline Negative & $63(70.8)$ & $65(73.0)$ & $70(78.7)$ \\
Positive & $26(29.2)$ & $24(27.1)$ & $19(21.3)$ \\
+ & $2(2.2)$ & $1(1.1)$ & $1(1.1)$ \\
++ & $3(3.4)$ & $4(4.4)$ & $1(1.1)$ \\
+++ & $12(13.5)$ & $14(15.7)$ & $7(7.9)$ \\
++++ & $9(10.1)$ & $5(5.6)$ & $10(11.2)$ \\
\hline
\end{tabular}

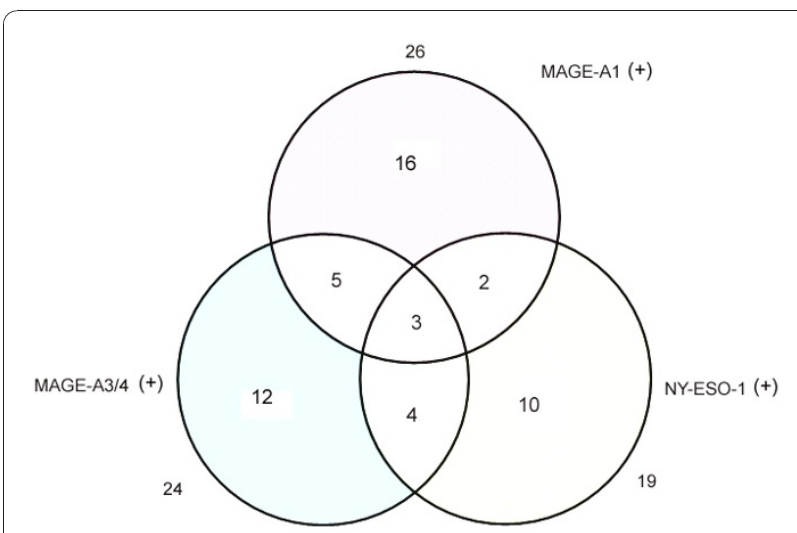

Figure 2 Venn diagram depicting the overlap in the expression of cancer-testis antigens in intrahepatic cholagiocarcinoma.

Patients with MAGE-A3/4 positive tumors had a significantly poorer outcome compared to those without MAGE-A3/4 expression. MAGE-A1 and NY-ESO-1 also demonstrated the same trend but did not reach statistical significance. Interestingly, negative expression in all CTAs correlated with a better prognosis than at least one CTAs expression, meanwhile, two or three CTAs expression had no impact on survival (Figure 3, Table 3). COX proportional hazard model analysis showed that at least one CTA expression was an independent prognostic indicator for IHCC, whereas the association of MAGE-A3/4

Table 2 Correlation between CTA expression pattern and HLA class I expression

\begin{tabular}{|c|c|c|c|}
\hline \multirow[t]{2}{*}{ CTA expression pattern } & \multicolumn{2}{|c|}{ HLA class I expression } & \multirow[t]{2}{*}{$P$ value } \\
\hline & $\begin{array}{l}\text { Positive } \\
(\mathrm{n}=51)\end{array}$ & $\begin{array}{l}\text { Down-regulated } \\
\quad(\mathrm{n}=38)\end{array}$ & \\
\hline \multicolumn{4}{|l|}{ MAGE-A1 } \\
\hline Positive & 18 & 8 & 0.144 \\
\hline Negative & 33 & 30 & \\
\hline \multicolumn{4}{|l|}{ MAGE-A3/4 } \\
\hline Positive & 11 & 13 & 0.184 \\
\hline Negative & 40 & 25 & \\
\hline \multicolumn{4}{|l|}{ NY-ESO-1 } \\
\hline Positive & 11 & 8 & 0.953 \\
\hline Negative & 40 & 30 & \\
\hline \multicolumn{4}{|l|}{1 CTA positive } \\
\hline With & 30 & 22 & 0.930 \\
\hline Without & 21 & 16 & \\
\hline \multicolumn{4}{|l|}{2 CTA positive } \\
\hline With & 9 & 5 & 0.565 \\
\hline Without & 42 & 33 & \\
\hline \multicolumn{4}{|l|}{3 CTA positive } \\
\hline With & 1 & 2 & 0.795 \\
\hline Without & 50 & 36 & \\
\hline
\end{tabular}


Table 3 Univariate analyses of prognostic factors associated with overall survival (OS)

\begin{tabular}{llll}
\hline Variable & Category & No. of patients & P \\
\hline Gender & female vs. male & 31 vs. 58 & 0.587 \\
Age & $<60$ vs. $\geq 60$, years & 19 vs. 70 & 0.532 \\
TNM stage & $1 / 2$ vs. $3 / 4$ & 34 vs. 55 & 0.007 \\
Tumor size & $\geq 5$ cm vs. $<5$ cm & 55 vs. 34 & 0.690 \\
Differentiation & well or mod vs. poor & 26 vs. 63 & 0.008 \\
Resection margin & R0 vs. R1/2 & 56 vs. 33 & 0.008 \\
Tumor number & single vs. multiple & 58 vs. 31 & 0.385 \\
Vascular invasion & with vs. without & 42 vs. 47 & 0.227 \\
Perineural invasion & with vs. without & 33 vs. 56 & 0.736 \\
Lymph node metastasis & with vs. without & 38 vs. 51 & 0.001 \\
Tumor recurrence & with vs. without & 47 vs. 42 & 0.022 \\
MAGE-A1 & Positive vs. negative & 26 vs. 63 & 0.116 \\
MAGE-A3/4 & Positive vs. negative & 24 vs. 65 & 0.009 \\
NY-ESO-1 & Positive vs. negative & 19 vs. 70 & 0.068 \\
1 CTA positive & with vs. without & 52 vs. 37 & 0.001 \\
2 CTA positive & with vs. without & 14 vs. 75 & 0.078 \\
3 CTA positive & with vs. without & 3 vs. 86 & 0.372 \\
\hline
\end{tabular}

with a shorter survival failed to persist in the multivariate analysis (Table 4).

\section{Discussion}

In this study, expression of three CTAs at protein level was investigated by immunohistochemistry. MAGE-A1,
Table 4 Multivariate analyses of factors associated with overall survival (OS)

\begin{tabular}{lllll}
\hline Variable & HR & \multicolumn{2}{c}{$\mathbf{9 5 \%}$ Confidence Interval } & P value \\
\cline { 3 - 4 } & & Lower & Upper & \\
\hline 1 CTA positive & 0.524 & 0.298 & 0.920 & 0.024 \\
MAGE-A3/4 & 0.897 & 0.505 & 1.594 & 0.711 \\
Differentiation & 0.447 & 0.263 & 0.758 & 0.003 \\
TNM stage & 1.122 & 0.597 & 2.110 & 0.721 \\
Lymph node metastasis & 0.389 & 0.207 & 0.732 & 0.003 \\
Tumor recurrence & 0.706 & 0.386 & 1.291 & 0.258 \\
Resection margin & 1.138 & 0.574 & 2.258 & 0.711
\end{tabular}

MAGE-A3/4 and NY-ESO-1 were selected considering that these antigens have been well-accredited and are being applied for clinical trials of vaccine immunotherapy [15-18]. The expression frequency of CTAs varies greatly in different tumors type $[19,20]$. Our results showed that expression rates of MAGE-A1, MAGE-A3/ 4 and NY-ESO-1 in IHCC were less than $30 \%$. According to the established criteria [21], IHCC should be classified to be low "CTA expressors". In a previous study, the expression rates of MAGE-A1, MAGE-A3 and NY-ESO-I in IHCC were 20.0\% (4/20), 20.0\% (4/20) and $10.0 \%(2 / 20)$ detected by RT-PCR [6]. However, in the immunohistochemical study by Tsuneyama et al. [7], 32 of 68 IHCC cases $(47.1 \%)$ demonstrated positive MAGE-A3 expression using a polyclonal antibody.

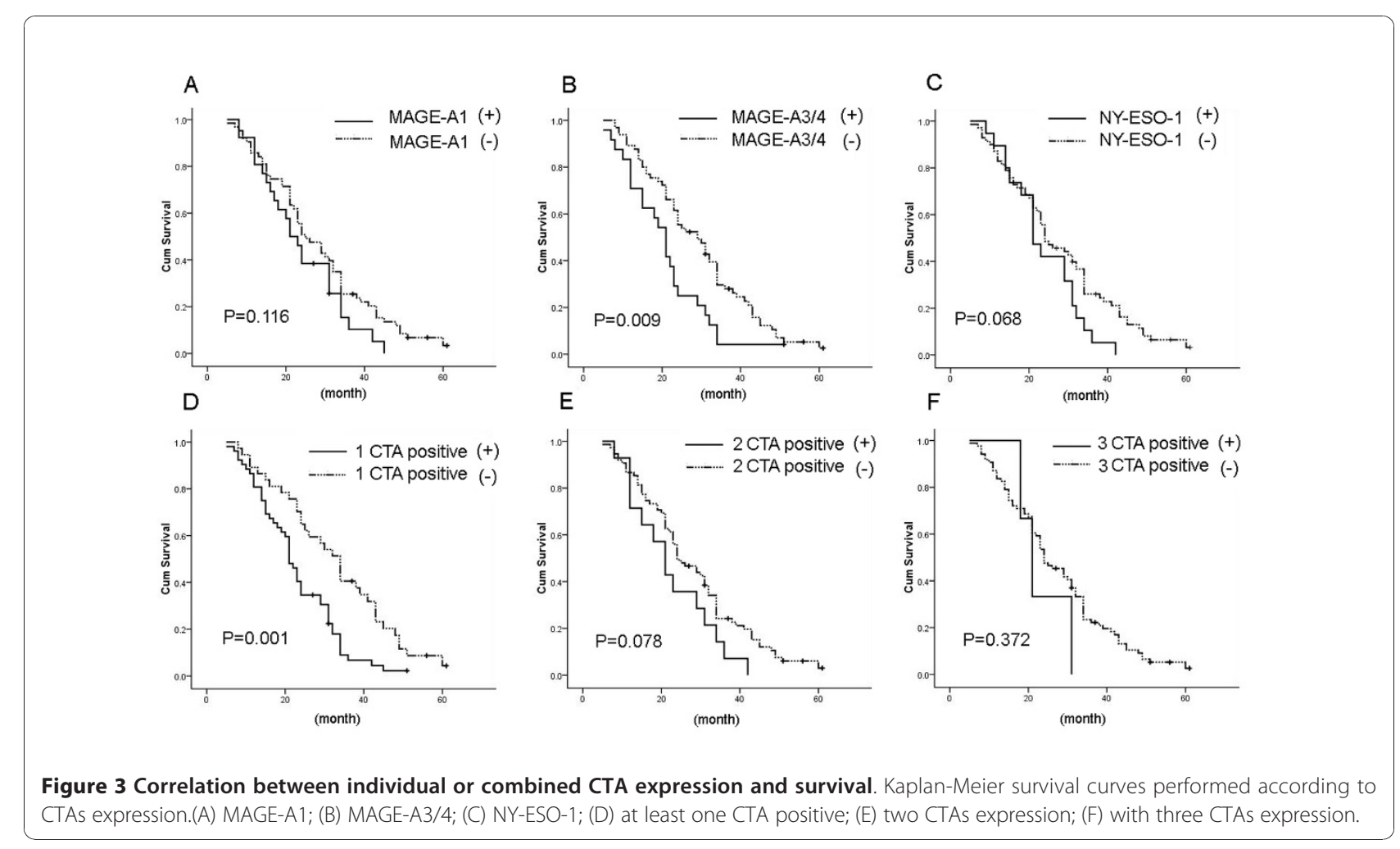


These discrepancies between our and previous studies may be related to the difference in the method of detection, the antibodies adopted and patient populations.

In this study, we also identified that only MAGE-3/4 and at least one positive CTA expression correlated aggressive phenotypes including bigger tumor size and higher recurrence rate. There was no other association observed between CTA markers (either individual or combined) with HLA class I expression and clinicopathological parameters of IHCC patients.

Curves of patients with positive for the individual or multiple CTAs (with two or three CTA positive) markers leaned towards a poorer outcome, however, only MAGEA3/4 reach statistical significance. We speculated that such statistically insignificant trends were likely to be due to the fact that only a small number of IHCC cases presented with positive CTA expression (either individual or co-expressed) in this study. Considering that combination of CTAs makers may reinforce the predictive value for prognosis and malignant phonotype by one single CTA alone, we next asked whether at least one CTA expression had $\mathrm{n}$ significant impact on outcome. We found that at least one CTA expression did indeed correlate with a significantly poorer survival. Furthermore, at least one positive CTA expression was also an independent prognostic factor for patients with IHCC.

Interestingly, in this study, MAGE-A1 and NY-ESO-1 positive IHCC tumors seem to have a relatively higher frequency of positive expression of HLA class I than MAGE-A3/4 positive cases. Recently, Kikuchi et al. [22] indicated that co-expression of CTA (XAGE-1b) and HLA class I expression may elicit a CD8+ T-cell response against minimal residual disease after surgery and resulted in prolonged survival of NSCLC patients, while expression of CTA combined with down-regulated HLA class I expression correlated with poor survival. Therefore, we speculated that a relatively high proportion of HLA Class I-negative cases in MAGE-A3/4 positive group may partly account for its association with significantly poor survival.

MAGE-A1, MAGE-A3/4 and NY-ESO-1 have been applied for clinical trials of vaccine immunotherapy for multiple cancer patients, but the utility of CTA immunotherapy against patients with IHCC remains investigated. In this study, using three CTA markers MAGE-A1, MAGE-A3/4 and NY-ESO-1, we identified a subgroup (58.4\%) of IHCC patients with at least one CTA expression having a poor prognosis. Moreover, high levels of expression of these antigens were observed in most positive cases. In our study, the concomitant expression of CTAs and HLA class I antigen was observed in $33.7 \%$ of the IHCC tumors, which indicating that it may be possible to immunise a significant proportion of IHCC patients with tumor-specific
CTLs. Based on our data, we suggest that a considerable number of IHCC patients at high-risk might benefit from specific immunotherapy targeted MAGE-A and NY-ESO-1.

This is the first study demonstrating a correlation between CTA and prognosis in IHCC. Furthermore, this present retrospective cohort study is limited to relatively small case series (although more than previous studies); therefore, further validation will be required before these antigens can be tested for targeted immunotherapy.

\section{Conclusion}

In conclusion, our data suggest that the cancer-testis antigens identified in this study might be novel biomarkers and therapeutic targets for patients with IHCC.

\section{Additional material}

Additional file 1: Table S1 Clinicopathological characteristics of patients included in this study. a table for the clinicaopathological characteristics of 89 IHCC patients.

\section{Acknowledgements}

This research was supported by grants from National Science Foundation of China (30772017, 30972730), Shanghai Municipal Commission for Science and Technology (08QH14001, 09JC1405400).

\section{Author details}

'Department of Hepatobiliary and Pancreatic Surgery, Henan Tumor Hospital, Zhengzhou, Henan 450008, PR China. ${ }^{2}$ Capital Medical University School of Stomatology, Beijing 100050, PR China. ${ }^{3}$ Changzheng Hospital, Second Military Medical University, Shanghai 200003, PR China.

\section{Authors' contributions}

JXZ and YL contributed to clinical data, samples collection immunohistochemistry analysis and manuscript writing. SXC and AMD were responsible for the study design and manuscript writing. All authors read and approved the final manuscript.

\section{Competing interests}

The authors declare that they have no competing interests.

Received: 8 November 2010 Accepted: 6 January 2011

Published: 6 January 2011

\section{References}

1. Patel $\mathrm{T}$ : Increasing incidence and mortality of primary intrahepatic cholangiocarcinoma in the United States. Hepatology 2001, 33:1353-1357.

2. Hsing AW, Gao YT, Han TQ, Rashid A, Sakoda LC, Wang BS, Shen MC, Zhang BH, Niwa S, Chen J, Fraumeni JF Jr: Gallstones and the risk of biliary tract cancer: a population-based study in China. Br J Cancer 2007, 97:1577-1582.

3. Suri A: Cancer testis antigens-their importance in immunotherapy and in the early detection of cancer. Expert Opin Biol Ther 2006, 6:379-389.

4. Toso JF, Oei C, Oshidari F, Tartaglia J, Paoletti E, Lyerly HK, Talib S, Weinhold KJ: MAGE-1-specific precursor cytotoxic T-lymphocytes present among tumor-infiltrating lymphocytes from a patient with breast cancer: characterization and antigen-specific activation. Cancer Res 1996, 56:16-20.

5. Caballero OL, Chen YT: Cancer/testis (CT) antigens: potential targets for immunotherapy. Cancer Sci 2009, 100:2014-2021.

6. Utsunomiya T, Inoue $H$, Tanaka F, Yamaguchi H, Ohta M, Okamoto M, Mimori K, Mori M: Expression of cancer-testis antigen (CTA) genes in intrahepatic cholangiocarcinoma. Ann Surg Oncol 2004, 11:934-940. 
7. Tsuneyama K, Sasaki M, Shimonishi T, Nakanuma Y: Expression of MAGE-A3 in intrahepatic cholangiocarcinoma and its precursor lesions. Pathol Int 2004, 54:181-186.

8. Jungbluth AA, Stockert $E$, Chen $\mathrm{YT}$, Kolb D, Iversen $\mathrm{K}$, Coplan $\mathrm{K}$, Williamson B, Altorki N, Busam KJ, Old L: Monoclonal antibody MA454 reveals a heterogeneous expression pattern of MAGE-1 antigen in formalin-fixed paraffin embedded lung tumours. $\mathrm{Br} J$ Cancer 2000, 83:493-497.

9. Hudolin T, Juretic A, Spagnoli GC, Pasini J, Bandic D, Heberer M, Kosicek M, Cacic M: Immunohistochemical expression of tumor antigens MAGE-A1, MAGE-A3/4, and NY-ESO-1 in cancerous and benign prostatic tissue. Prostate 2006, 66:13-18.

10. Gjerstorff MF, Kock K, Nielsen O, Ditzel HJ: MAGE-A1, GAGE and NY-ESO-1 cancer/testis antigen expression during human gonadal development. Hum Reprod 2007, 22:953-960.

11. Rimoldi D, Salvi S, Schultz-Thater E, Spagnoli GC, Cerottini JC: Anti-MAGE-3 antibody 57B and anti-MAGE-1 antibody $6 \mathrm{C} 1$ can be used to study different proteins of the MAGE-A family. Int I Cancer 2000, 86:749-51.

12. Landry C, Brasseur F, Spagnoli GC, Marbaix E, Boon T, Coulie P, Godelaine D: Monoclonal antibody 57B stains tumor tissues that express gene MAGEA4. Int J Cancer 2000, 86:835-841.

13. Kikuchi E, Yamazaki K, Torigoe T, Cho Y, Miyamoto M, Oizumi S, Hommura F, Dosaka-Akita H, Nishimura M: HLA class I antigen expression is associated with a favorable prognosis in early stage non-small cell lung cancer. Cancer Sci 2007, 98:1424-1430.

14. Perez D, Herrmann T, Jungbluth AA, Samartzis P, Spagnoli G, Demartines N, Clavien PA, Marino S, Seifert B, Jaeger D: Cancer testis antigen expression in gastrointestinal stromal tumors: new markers for early recurrence. Int $J$ Cancer 2008, 123:1551-1555.

15. Tyagi P, Mirakhur B: MAGRIT: the largest-ever phase III lung cancer trial aims to establish a novel tumor-specific approach to therapy. Clin Lung Cancer 2009, 10:371-374.

16. Bender A, Karbach J, Neumann A, Jäger D, Al-Batran SE, Atmaca A, Weidmann E, Biskamp M, Gnjatic S, Pan L, Hoffman E, Old LJ, Knuth A, Jäger E: LUD 00-009: phase 1 study of intensive course immunization with NY-ESO-1 peptides in HLA-A2 positive patients with NY-ESO-1expressing cancer. Cancer Immun 2007, 19:7-16.

17. Shigematsu Y, Hanagiri T, Shiota H, Kuroda K, Baba T, Mizukami M, So T, Ichiki Y, Yasuda M, So T, Takenoyama M, Yasumoto K: Clinical significance of cancer/testis antigens expression in patients with non-small cell lung cancer. Lung Cancer 2010, 68:105-110.

18. Weide B, Pascolo S, Scheel B, Derhovanessian E, Pflugfelder A, Eigentler TK, Pawelec G, Hoerr I, Rammensee HG, Garbe CJ: Direct injection of protamine-protected mRNA: results of a phase $1 / 2$ vaccination trial in metastatic melanoma patients. Immunother 2009, 32:498-507.

19. Sadanaga N, Nagashima H, Tahara K, Yoshikawa Y, Mori M: The heterogeneous expression of MAGE-3 protein: difference between primary lesions and metastatic lymph nodes in gastric carcinoma. Oncol Rep 1999, 6:975-977.

20. Scanlan MJ, Simpson AJ, Old L: The cancer/testis genes: review, standardization, and commentary. Cancer Immun 2004, 4:1.

21. Grizzi F, Franceschini B, Hamrick C, Frezza EE, Cobos E, Chiriva-Internati M: Usefulness of cancer-testis antigens as biomarkers for the diagnosis and treatment of hepatocellular carcinoma. J Transl Med 2007, 5:3.

22. Kikuchi E, Yamazaki K, Nakayama E, Sato S, Uenaka A, Yamada N, Oizumi S, Dosaka-Akita H, Nishimura M: Prolonged survival of patients with lung adenocarcinoma expressing XAGE-1b and HLA class I antigens. Cancer Immun 2008, 8:13.

doi:10.1186/1756-9966-30-2

Cite this article as: Zhou et al:: Expression and prognostic significance

of cancer-testis antigens (CTA) in intrahepatic cholagiocarcinoma.

Journal of Experimental \& Clinical Cancer Research 2011 30:2.

\section{Submit your next manuscript to BioMed Central and take full advantage of:}

- Convenient online submission

- Thorough peer review

- No space constraints or color figure charges

- Immediate publication on acceptance

- Inclusion in PubMed, CAS, Scopus and Google Scholar

- Research which is freely available for redistribution

Submit your manuscript at www.biomedcentral.com/submit 